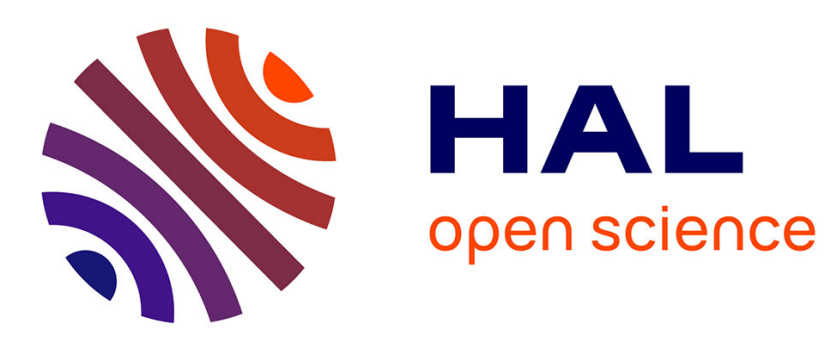

\title{
Invariance Properties of Inviscid Fluids of Grade $\mathbf{n}$ Pierre Casal, Henri Gouin
}

\section{To cite this version:}

Pierre Casal, Henri Gouin. Invariance Properties of Inviscid Fluids of Grade n. Lecture Notes in Physics, 1989, 344, pp.85-98. 10.1007/BFb0024930 . hal-00266215

\section{HAL Id: hal-00266215 https://hal.science/hal-00266215}

Submitted on 21 Mar 2008

HAL is a multi-disciplinary open access archive for the deposit and dissemination of scientific research documents, whether they are published or not. The documents may come from teaching and research institutions in France or abroad, or from public or private research centers.
L'archive ouverte pluridisciplinaire HAL, est destinée au dépôt et à la diffusion de documents scientifiques de niveau recherche, publiés ou non, émanant des établissements d'enseignement et de recherche français ou étrangers, des laboratoires publics ou privés. 


\title{
Invariance Properties of Inviscid Fluids of Grade $n$
}

\author{
Pierre Casal and Henri Gouin ${ }^{2}$ \\ ${ }^{a}$ University of Aix-Marseille \& C.N.R.S. U.M.R. 6181 \\ Av. Escadrille Normandie-Niemen, Box 322, 13397 Marseille Cedex 20, France. \\ Revisited version in memory of Professor Pierre Casal
}

\begin{abstract}
Fluids of grade $\mathrm{n}$ are continuous media in dynamic changes of phases avoiding the surfaces of discontinuity and representing the capillary layers in liquid-vapour interfaces. We recall the thermodynamic form of the equation of motion for inviscid fluids of grade $\mathrm{n}$ [1]. First integrals and theorems of circulation are deduced. A general classification of flows is proposed.
\end{abstract}

Key words: Inviscid fluids; invariance properties of flows; classification of flows; complex fluids.

PACS: 47.10.-g; 47.10.ab; 47.15.ki

$1991 \mathrm{MSC}: 76 \mathrm{~A} 02 ; 76 \mathrm{M} 60$

\section{Introduction}

In continuum mechanics, media of first gradient order cannot represent fluids with strong density variations. Material surfaces need their own characteristic behaviour and energy properties [2].

Fluids of grade $n$ ( $n$ is any integer greater than 1 ) are continuous media with an internal energy per unit mass $\varepsilon$ which is a function of the entropy $s$, the density $\rho$ and spatial gradients up to the $n-1$ order:

$$
\varepsilon=\varepsilon\left(s, \operatorname{grad} s, \ldots,(\operatorname{grad})^{(n-1)} s, \rho, \operatorname{grad} \rho, \ldots,(\operatorname{grad})^{(n-1)} \rho\right)
$$

Email address: (Corresponding author) henri.gouin@univ-cezanne.fr (Henri Gouin).

Preprint submitted to Lecture Notes in Physics, vol. 344, p. 85-98, Springer, Berlin (1989) 
D.J. Korteweg pointed out the advantage of fluids of grade $n$ with respect to the density and removed the discontinuity surfaces representing the capillary layers in liquid-vapour interfaces [3].

We improve the model accuracy by taking into account the successive gradients of density. Recently, this approach was used in the case of dynamic changes of phase [4]. Till now, the thermodynamic part of the fluid has been neglected. Due to the fact it is not possible to consider a virtual displacement of temperature, it is necessary to use the entropy by the way of the internal energy.

The equation of motion with both strong gradients of density and entropy is obtained. One can accord importance to the fact that the thermodynamic form of the equation of motion is independent of the order $n$. Flows with strong variations of temperature such as those associated with combustion phenomena or non-isothermal interfaces may be considered.

The conservative flows of classical perfect fluids are only a mathematical model. It is also the case for fluids of grade $n$. The study of their structure is necessary. The conclusions for fluids of grade $n$ are of the same kind as for compressible fluid [5]: it is possible to obtain a Clebsh transformation of the motion equations (potential equations) and many invariance properties and first integrals. The potential equations may be used to classify motions in the same manner as in the case of perfect fluids [6,7].

The simplest case is for fluids of grad 2 (or thermocapillary fluids): the internal energy is a function only of the density, the entropy and their first spatial derivatives

$$
\varepsilon=\varepsilon(s, \operatorname{grad} s, \rho, \operatorname{grad} \rho)
$$

and $n>2$ adds little except for complications in the momentum balance equation. If we consider flows through liquid-vapour interfaces and use convenient physical units, the equations of thermocapillary fluids are able to study non-isothermal motions and yield a possible interpretation of film boiling phenomena $[8,9]$.

\section{Thermodynamic form of the equation of motion for inviscid flu- ids of grade $\mathbf{n}$}

The principle of virtual works allows to obtain the equation of motion. In the case of a perfect fluid motion, it is written by means of the Hamilton principle $[5,6,10]$.

The variations of particle motions are deduced from families of virtual motions of the fluid in the form [5]

$$
\mathbf{X}=\Psi(\mathbf{x}, t, \alpha)
$$


where $\alpha$ is a parameter defined in a neighbourhood of zero. The real motion corresponds to $\alpha=0$.

A particle is represented in Lagrange coordinates by $\mathbf{X}\left(X^{1}, X^{2}, X^{3}\right)$ as a position in a reference space $\mathcal{D}_{o}$. At time $t$ its position is given in $\mathcal{D}_{t}$ by the Eulerian representation $\mathbf{x}\left(x^{1}, x^{2}, x^{3}\right)$ (see Appendix). The virtual displacements associated with a variation of the real motion can be written

$$
\delta \mathbf{X}=\left.\frac{\partial \Psi}{\partial \alpha}(\mathbf{x}, t, \alpha)\right|_{\alpha=0}
$$

The variation is dual with Serrin's [5]: the two variations are mathematically equivalent [11]. The Lagrangian of a fluid of $\operatorname{grad} n$ is defined as

$$
L=\rho\left(\frac{1}{2} \mathbf{V}^{*} \mathbf{V}-\varepsilon-\Omega\right),
$$

where $\mathbf{V}$ denotes the velocity, $\Omega$ denotes the extraneous force potential defined in $\mathcal{D}_{t}$ and ${ }^{*}$ is the transposition in $\mathcal{D}_{t}$ such that $\mathbf{V}^{*} \mathbf{V}$ is the scalar product of $\mathbf{V}$ by $\mathbf{V}$. Between times $t_{1}$ and time $t_{2}$, the Hamiltonian action is

$$
a=\int_{t_{1}}^{t_{2}} \int_{D_{t}} L d v d t
$$

The density verifies the balance equation

$$
\rho \operatorname{det} F=\rho_{o}(\mathbf{X})
$$

where $\rho_{o}$ is defined in $\mathcal{D}_{o}$ and $F$ is the deformation gradient. We deduce

$$
\frac{d \rho}{d t}+\rho \operatorname{div} \mathbf{V}=0
$$

The entropy variation is the sum of a variation associated with the virtual motion and another one, $\delta_{1} s$, related to the particle [9]

$$
\delta s=\left(\frac{\partial s}{\partial \mathbf{X}}\right) \delta \mathbf{X}+\delta_{1} s,
$$

where $\delta_{1} s$ and $\delta \mathbf{X}$ are independent. To obtain the equation of motion, we consider the conservative case corresponding to $\delta_{1} s=0$.

Classical methods of variational calculus provide the Hamiltonian action variation

$$
\delta a=0
$$

A calculus as in [1] yields

$$
\delta a=\int_{t_{1}}^{t_{2}} \int_{\mathcal{D}_{t}} \rho_{o}\left[\frac{d}{d t}\left(\mathbf{V}^{*} F\right)-\theta \operatorname{grad}_{\mathrm{o}}^{*} s-\operatorname{grad}_{\mathrm{o}}^{*} m\right] \delta \mathbf{X} d v_{o} d t
$$


where

$$
\left\{\begin{array}{l}
m=\frac{1}{2} \mathbf{V}^{*} \mathbf{V}-h-\Omega \\
h=\varepsilon+\frac{p}{\rho} \\
p=\rho^{2} \varepsilon_{\rho}^{\prime}+\rho \sum_{k=1}^{n-1}(-1)^{k} \operatorname{div}(k)\left(\rho \frac{\partial \varepsilon}{\partial\left(\operatorname{grad}^{(k)} \rho\right)}\right) \\
\theta=\varepsilon_{s}^{\prime}+\frac{1}{\rho} \sum_{k=1}^{n-1}(-1)^{k} \operatorname{div}(k)\left(\rho \frac{\partial \varepsilon}{\partial\left(\operatorname{grad}^{(k)} s\right)}\right)
\end{array}\right.
$$

We have taken into account the relation $\delta \rho=\rho \operatorname{div}_{\mathrm{o}}(\delta \mathbf{X})+\frac{1}{\operatorname{det} F} \frac{\partial \rho_{o}}{\partial \mathbf{X}} \delta \mathbf{X}$ and $\delta \mathbf{V}=-F \frac{d(\delta \mathbf{X})}{d t} ; \operatorname{div}^{(k)}$ and $\operatorname{grad}^{(k)}$ are the divergence and the gradient operators in $\mathcal{D}_{t}$ reiterated $k$-times; $\operatorname{div}_{\mathrm{o}}$ and $\operatorname{grad}_{\mathrm{o}}$ are the divergence and the gradient operators in $\mathcal{D}_{o}$.

Obviously, $p, \theta$ and $h$ have respectively the dimension of a pressure, a temperature and a specific enthalpy. We call these quantities the pressure, temperature and enthalpy of the fluid of grade $n$.

The Hamilton principle yields:

For any displacement $\delta \mathbf{X}$ null on the edge of $\mathcal{D}_{o}, \delta a=0$.

We get the equation of motion

$$
\frac{d\left(\mathbf{V}^{*} F\right)}{d t}-\theta \operatorname{grad}_{\mathrm{o}}^{*} \mathrm{~s}-\operatorname{grad}_{\mathrm{o}}^{*} \mathrm{~m}=0
$$

Let us note that $\left(\boldsymbol{\Gamma}^{*}+\mathbf{V}^{*} \frac{\partial \mathbf{V}}{\partial \mathbf{x}}\right) F=\frac{d\left(\mathbf{V}^{*} F\right)}{d t}$, we obtain

$$
\Gamma=\theta \operatorname{grad} s-\operatorname{grad}(h+\Omega)
$$

In case of inviscid thermocapillary fluids the dissipative function is null and the equations of motion and energy imply [12]:

$$
\rho \theta \frac{d s}{d t}+\operatorname{div} \mathbf{q}-r=0
$$

where $\mathbf{q}$ is the heat flux vector and $r$ the heat supply. This result is extended to the fluids of grade $\mathrm{n}[13]$.

A constitutive equation must be added to these equations, which yields the behaviour of the heat flux vector q. For example, the Fourier law is

$$
\mathbf{q}=-k \operatorname{grad} \theta \text { and } k \geq 0
$$


The heat supply is assumed to be given in similar way than the extraneous force potential. From the heat conduction inequality, we obtain the ClausiusDuhem inequality and the fluids of grade $n$ are compatible with the second law of thermodynamics $[14,15]$

$$
\rho \frac{d s}{d t}+\operatorname{div}\left(\frac{\mathbf{q}}{\theta}\right)-\frac{\mathrm{r}}{\theta} \geq 0
$$

Consequently, the equations of motions of inviscid fluids of grade $\mathrm{n}$ verify

$$
\left\{\begin{array}{l}
\boldsymbol{\Gamma}=\theta \operatorname{grad} s-\operatorname{grad}(h+\Omega) \\
\frac{d \rho}{d t}+\rho \operatorname{div} \mathbf{V}=0 \\
\rho \theta \frac{d s}{d t}+\operatorname{div} \mathbf{q}-\mathrm{r}=0 \\
\mathbf{q}=-k \operatorname{grad} \theta \text { and } k \geq 0
\end{array}\right.
$$

The thermodynamic form of the motion equation of inviscid fluids is equivalent to the classic balance equation. The thermodynamic form is the same for all fluids (independently of the order $n$ ). Nevertheless, the complexity of balance equations increases with $n$. In fact, only the expressions for the temperature and the enthalpy become more complex and we have to modify the definitions of these quantities ${ }^{1}$.

\section{Thermodynamic hypothesis on the motion}

If we study the invariance properties of the motions, we must assume that the heat supply allows a behaviour expressing that a thermodynamic quantity $T$ has a zero material derivative or - with a stronger assumption - is constant everywhere in the flow. Consequently equations (4) and (5) are replaced by one of the two conditions

$$
\frac{d T}{d t}=0 \quad \text { or } \quad T=C^{\text {ste }}
$$

For example, we will consider the case of an isentropic motion $(T=s)$. Then,

$$
\frac{d s}{d t}=0
$$

For the case of an isothermal motion $(T=\theta)$. Then,

$$
\theta=C^{\text {ste }}
$$

$\overline{1}$ The definitions are given by system (2). 
They are two limit cases: one is associated with fast motions, the other with slow motions ${ }^{2}$. Then, the fluid motions satisfy the system consisting of equations (1), (3) and (7).

We consider the more general case when $T$ is a differentiable function of $\theta$ and $s$ such that $\frac{\partial T}{\partial \theta} \neq 0$. Consequently, we denote by $H(T, s)$ a differentiable function of the variables $T$ and $s$ such that $\frac{\partial H}{\partial s}=\theta(T, s)$.

The function $H$ is defined, to an unknown function of $T$, by the differential form

$$
d H=\theta d s-u d T
$$

The two variables $u$ and $T$ can be used instead of $\theta$ and $s$ to study the general case by transforming Eq. (3) following the method of Lemma 1. Let us denote $g=h-H$, Eq. (3) can be written:

$$
\Gamma=u \operatorname{grad} \mathrm{T}-\operatorname{grad}(\mathrm{g}+\Omega)
$$

Let us note that if $T=\theta, g$ is the free enthalpy $(g=h-\theta s)$ and $u=-s$, and the motions satisfy System $((1),(7),(8))$.

First integrals are obtained by the research of invariant scalars as the state variables of the fluid (the density, the entropy, the velocity, with a zero material derivative) and expression (8) allows us to make a single classification for the fluids.

The momentum equation expresses the balance of forces (inertial forces, body forces and stresses).

Let us note that System $((1),(7),(8))$ is not in balance form, but expresses the fluid evolution: the relation $\frac{d T}{d t}=0$ implies $T$ is constant along trajectory and the equation of continuity $\frac{d \rho}{d t}=-\rho \operatorname{div} \mathbf{V}$ yields the density variation. It will be shown that equations (3) or (8) describe the evolution of the velocity field.

2 The Euler equation represents the equation of motion for classical inviscid fluids. Such motions can be obtained with non-negligible heat flux and heat supply. They correspond to physical situations. As a matter of fact, in the non-dimensional equation of motion for viscous fluids [16,17], it is often justified to neglect the viscous terms when, with respect to other physical quantities, the Reynolds number is large. Nevertheless, for large temperature gradients and with a Prandlt number of order one, it is not possible to remove the terms of thermal conductivity. Such a case arises when we study the "thermal" boundary layer $[18,19]$ and slow motions of natural convection in fluid with gravity and heat sources associated with large differences of temperature [20]. 


\section{Material and convective derivatives}

The material derivative of any tensorial quantity expressed in the physical space is the derivative with respect to time when one follows the particle along the motion.

The tensorial quantity has an image in the reference space. The image in the physical space of the derivative with respect to time of the tensorial quantity in the reference space is the value of the convective derivative. The convective derivative is denoted by $d_{c}$ (see Appendix).

The material and the convective derivatives of a scalar are equal. A tensor is convected by the flow when its convective derivative is null.

If we transpose Eq. (8), we get the convective derivative of the velocity covector $\mathbf{V}^{*}$ in the form

$$
d_{c} \mathbf{V}^{*}=\operatorname{grad}^{*}\left(\frac{1}{2} \mathbf{V}^{2}-\mathrm{g}-\Omega\right)+\mathrm{u} \operatorname{grad}^{*} \mathrm{~T} .
$$

Eq. (9) is equivalent to Eq. (8) and yields the Helmholtz-Kelvin theorem in its most general form:

Let us consider an arbitrary fluid curve $\mathcal{C}$ such that $I(t)=\int_{\mathcal{C}} \mathbf{V}^{*} d \mathbf{x}$ is the circulation of the velocity field. The circulation of the convective derivative of the velocity field is

$$
\frac{d I}{d t}=\int_{\mathcal{C}} d_{c} \mathbf{V}^{*} d \mathbf{x}=\int_{\mathcal{C}} d\left(\frac{1}{2} \mathbf{V}^{2}-g-\Omega\right)+\int_{\mathcal{C}} u d T
$$

If $\mathcal{C}$ is a closed curve, the integral $\int_{\mathcal{C}} d\left(\frac{1}{2} \mathbf{V}^{2}-g-\Omega\right)$ is null. Moreover, if $\mathcal{C}$ is located on a $T$-surface (surfaces $T=C^{\text {ste }}$ are fluid surfaces and this assumption is compatible with the fact that $\mathcal{C}$ is a fluid curve), the second integral is null and $I$ is constant along the motion.

When the entropy is constant all over the fluid $\left(T=s=C^{s t e}\right)$, the so-called Kelvin theorem corresponds to the special case of homentropic motions: the circulation of the velocity field on any closed curve is constant.

Due to Noether's theorem, it is known that any conservation law can be represented by a group of invariance. It has been shown the conservation law expressed by Helmholtz-Kelvin theorems associated with isentropic fluid curves corresponds to the group of the permutations of the particles of equal entropy $[21,22]$. This group keeps the equations of motion invariant for both a classical perfect fluid and a perfect fluid of grade $n$. We propose to define a general perfect fluid as a fluid invariant by this group or as a continuous medium whose motions are submitted to Helmholtz-Kelvin theorems. 


\section{$5 \quad$ Potential equations}

Let $\varphi$ and $\psi$ be two scalar fields such that the material derivatives verify

$$
\frac{d \varphi}{d t}=\frac{1}{2} \mathbf{V}^{2}-g-\Omega \text { and } \frac{d \psi}{d t}=u
$$

Equation (9) is equivalent to

$$
d_{c} \mathbf{V}^{*}=d_{c}\left(\operatorname{grad}^{*} \varphi+\psi \operatorname{grad}^{*} \mathrm{~T}\right)
$$

The covector $\mathbf{V}^{*}$ and the covector $\mathbf{W}^{*}=\operatorname{grad}^{*} \varphi+\psi \operatorname{grad}^{*} \mathrm{~T}$ have the same convective derivative. The difference $(\mathbf{V}-\mathbf{W})^{*}$ is a covector convected by the flow, which leads to the system

$$
\left\{\begin{aligned}
\mathbf{V} & =\operatorname{grad} \varphi+\psi \operatorname{grad} \mathrm{T}+\mathbf{C} \\
\frac{d T}{d t}=0, \quad \frac{d \varphi}{d t} & =\frac{1}{2} \mathbf{V}^{2}-g-\Omega, \quad \frac{d \psi}{d t}=u, \quad d_{c} \mathbf{C}^{*}=0
\end{aligned}\right.
$$

System $((1),(7),(8))$ is equivalent to system $((1),(10))$.

In this case, the generalized Helmholtz-Kelvin theorem is reduced to the circulation of $\mathbf{C}$ along any fluid curve is constant.

Due to the Stokes formulae, the vector $\mathbf{U}=\frac{1}{\rho} \operatorname{rot} \mathbf{C}$ is convected by the flow $\left(d_{c} \mathbf{U}=0\right)$ i.e. :

$$
\frac{d \mathbf{U}}{d t}-\frac{\partial \mathbf{V}}{\partial x} \mathbf{U}=0
$$

Due to the fact that $\mathbf{C}=\gamma \operatorname{grad} \nu$, where $\gamma$ and $\nu$ are two scalars convected by the flow and $\operatorname{grad} \varphi, \operatorname{grad} \psi, \operatorname{grad} \nu$ are three independent vectors (see Lemma 2 and Theorem 3), the system (10) leads to the system of potential equations

$$
\left\{\begin{array}{c}
\mathbf{V}=\operatorname{grad} \varphi+\psi \operatorname{grad} T+\gamma \operatorname{grad} \nu \\
\frac{d T}{d t}=0, \quad \frac{d \varphi}{d t}=\frac{1}{2} \mathbf{V}^{2}-g-\Omega, \quad \frac{d \psi}{d t}=u, \quad \frac{d \gamma}{d t}=0, \quad \frac{d \nu}{d t}=0
\end{array}\right.
$$

In the general case, the velocity field is associated with five potentials; $T$ is one of them ${ }^{3}$.

$\overline{3}$ The velocity field depends also on the density which is not considered as a potential. 


\section{Classification of motions}

As for $T$, the potentials $\gamma$ and $\nu$ have a zero material derivative. They are first integrals of the motion [23].

These potentials have constant values on each trajectory; when they are constant in a fluid domain, the values remain constant in time-space.

In the domain convected by the flow, the fluid velocity is expressed by fewer potentials characterizing the kinematic properties of the motion. In an adjoining fluid domain, the motion may be different. Then, the flow is separated into different domains that move, remain adjacent and keep their own characteristics.

Due to the absence of viscosity, in hydrodynamics of incompressible perfect fluids the flow is divided into vortical and irrotational domains that do not mix. The following study shows that, for all inviscid fluids of any grade (compressible or not), the vortical domain may be divided into several parts of different kinds ${ }^{4}$

First integrals are tensorial quantities of different nature. For a non-scalar tensor, the first integral is a tensor convected by the flow (or with a zero convective derivative) i.e. has a constant tensorial image in a reference space.

\subsection{Oligotropic motions}

When the covector $\mathbf{C}^{*}$ is zero (i.e. $\gamma=0$ or $\nu=C^{\text {ste }}$.), the velocity field has a particular kinematic type. We have the following properties:

(a) The velocity field can be expressed by the use of three potentials and the equations of motion are

$$
\left\{\begin{array}{c}
\mathbf{V}=\operatorname{grad} \varphi+\psi \operatorname{grad} T \\
\frac{d T}{d t}=0, \frac{d \varphi}{d t}=\frac{1}{2} \mathbf{V}^{2}-g-\Omega, \frac{d \psi}{d t}=u .
\end{array}\right.
$$

(b) From System (13) we get

$$
\operatorname{rot} \mathbf{V}=\operatorname{grad} \psi \wedge \operatorname{grad} T
$$

then

$$
\operatorname{grad}^{*} T \operatorname{rot} \mathbf{V}=0
$$

$\overline{4}$ System (12) assumes the flow to be without discontinuity. A shock wave transforms the nature of flow $[11,24]$. 
Consequently, the vortex lines are located on the surfaces $T=C^{\text {ste }}$.

Property (14) is equivalent to System (13). The motions, we call oligotropic motions, are intermediate between irrotational and general motions. In System (13), the expression of the velocity is independent of time and the motion remains oligotropic at any moment.

\subsection{Homentropic motions}

The thermodynamic condition is $T=C^{\text {ste }}$ (which is stronger than $\frac{d T}{d t}=0$ ) and the velocity field is of another particular kind. When $T=s$, the motion is not only isentropic but also homentropic. We have the following properties:

(a) The velocity field can be expressed with the use of three potentials and the equations of motion are

$$
\left\{\begin{array}{c}
\mathbf{V}=\operatorname{grad} \varphi+\psi \operatorname{grad} \nu \\
\frac{d T}{d t}=0, \frac{d \varphi}{d t}=\frac{1}{2} \mathbf{V}^{2}-g-\Omega, \frac{d \gamma}{d t}=0, \frac{d \nu}{d t}=0
\end{array}\right.
$$

In fact the Clebsch representation [16] is practically worthless because the curves $\gamma=C^{\text {ste }}$ and $\nu=C^{\text {ste }}$ are the vortical lines whose complexity is well known [25]. It is easier to write the motion equations in an equivalent form:

$$
\left\{\begin{array}{c}
\mathbf{V}=\operatorname{grad} \varphi+\mathbf{C} \\
\frac{d T}{d t}=0, \quad \frac{d \varphi}{d t}=\frac{1}{2} \mathbf{V}^{2}-g-\Omega, \quad d_{c} \mathbf{C}^{*}=0 .
\end{array}\right.
$$

(b) Vector $\frac{1}{\rho} \operatorname{rot} \mathbf{V}$ is a convected vector

$$
d_{c}\left(\frac{1}{\rho} \operatorname{rot} \mathbf{V}\right)=0
$$

When a thermodynamic potential $T$ is constant in the flow, the Cauchy theorem for homentropic motions is generalized and Eq. (15) is characteristic of these motions. 


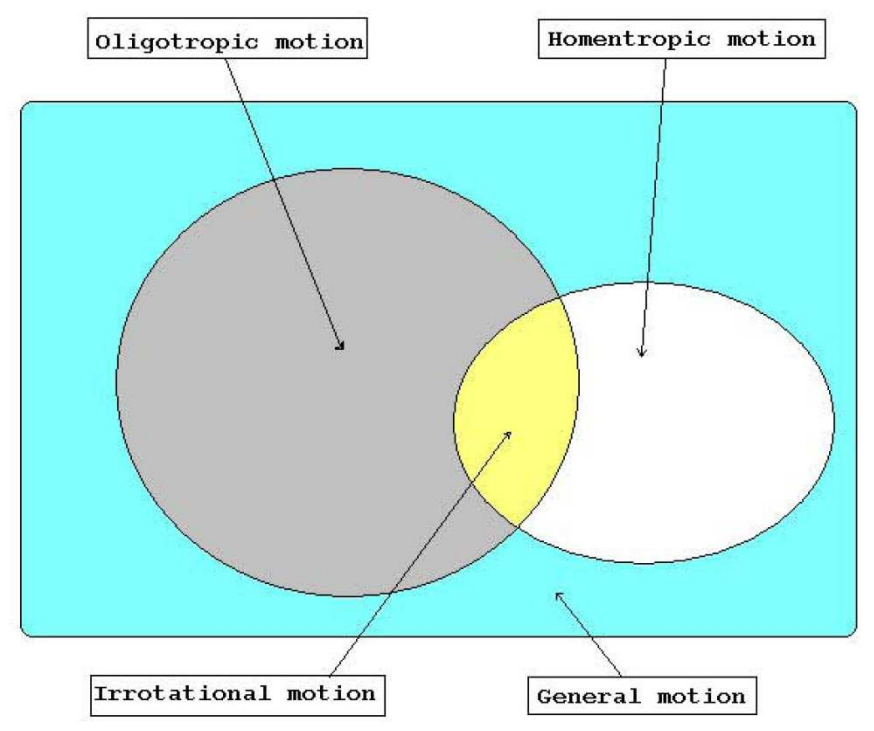

Fig. 1. For perfect fluid motions, the fluid domains are distributed in four parts. Convected by the flow, the domains become deformed but do not mix.

\subsection{Irrotational motion}

If an oligotropic motion is a motion where $T$ is constant, the velocity field derives from only one potential

$$
\left\{\begin{array}{l}
\mathbf{V}=\operatorname{grad} \varphi \\
\frac{d \varphi}{d t}=\frac{1}{2} \mathbf{V}^{2}-g-\Omega
\end{array}\right.
$$

At every time, $\operatorname{rot} \mathbf{V}=0{ }^{5}$. This is the case of irrotational motions: the velocity whose the evolution corresponds to the equation of Bernoulli is the gradient of a single potential.

\subsection{Permanent motions}

For a permanent motion, Equation (8) may be written in a generalized CroccoVaszonyi form

$$
\operatorname{rot} \mathbf{V} \wedge \mathbf{V}=u \operatorname{grad} T-\operatorname{grad} G
$$

where $G=\frac{1}{2} \mathbf{V}^{2}+g+\Omega$.

If $T=s, g$ is the specific enthalpy and $G$ the Lamb function [16] (surfaces

$\overline{5}$ When at one moment $\operatorname{rot} \mathbf{V}=0$, this property is not necessarily verified at every time as in hydrodynamics of compressible fluids. 
where $G=C^{\text {ste }}$ are Lamb surfaces). Consequently,

$$
\frac{d T}{d t}=0 \quad \Leftrightarrow \quad \frac{d G}{d t}=0
$$

$G$ is a scalar first integral of the motion and we use $G$ for function $\nu$. Potential equations for general permanent flows are

$$
\left\{\begin{array}{c}
\mathbf{V}=\operatorname{grad} \varphi_{1}+\psi_{1} \operatorname{grad} T+\gamma_{1} \operatorname{grad} G \\
\frac{d \varphi_{1}}{d t}=\frac{1}{2} \mathbf{V}^{2}-g-\Omega, \frac{d \psi}{d t}=u, \frac{d T}{d t}=0, \frac{d \gamma_{1}}{d t}=0, \frac{d G}{d t}=0 .
\end{array}\right.
$$

Let us note: $\frac{d T}{d t}=0$ and $\frac{d G}{d t}=0 \Rightarrow \frac{\partial \varphi_{1}}{\partial t}=-G$; if $\varphi=\varphi_{1}+t G, \varphi$ is independent of time. Let us denote $\gamma_{1}=\gamma+t$ and $\psi_{1}=\psi$, the equations of motion can be written with potentials independent of time

$$
\left\{\begin{aligned}
\mathbf{V} & =\operatorname{grad} \varphi+\psi \operatorname{grad} T+\gamma \operatorname{grad} G \\
\frac{d \psi}{d t} & =u, \frac{d T}{d t}=0, \frac{d \gamma}{d t}=-1, \frac{d G}{d t}=0 .
\end{aligned}\right.
$$

Let us note: $\mathbf{C}=(\gamma+t) \operatorname{grad} G$ and $d_{c} \mathbf{C}^{*}=0$; the behaviour of $\varphi\left(\frac{d \varphi}{d t}=\mathbf{V}^{2}\right)$ is a consequence of the previous equations.

The two scalars $T$ and $G$ characterize the motion. The classification of motions is in accordance with the respective positions of the surfaces $T=C^{\text {ste }}$ and $G=C^{\text {ste }}$ (when $T=s$ they are the isentropic and the Lamb surfaces). We have different cases

(a) General case: $T$ and $G$ are independent $(\operatorname{or} \operatorname{grad} T \wedge \operatorname{grad} G \neq 0)$.

The $T$-surfaces (isentropic surfaces) and the $G$-surfaces (Lamb surfaces) are different.

(b) $T$ is not constant and $G=G(T)$ : the $T$-surfaces are $G$-surfaces.

Replacing $\psi$ par $\psi+\gamma G_{T}^{\prime}$ we get

$$
\left\{\begin{array}{l}
\mathbf{V}=\operatorname{grad} \varphi+\psi \operatorname{grad} T \\
\frac{d \psi}{d t}=u-G^{\prime}(T), \frac{d T}{d t}=0 .
\end{array}\right.
$$

These equations characterize permanent oligotropic motions [6]. 


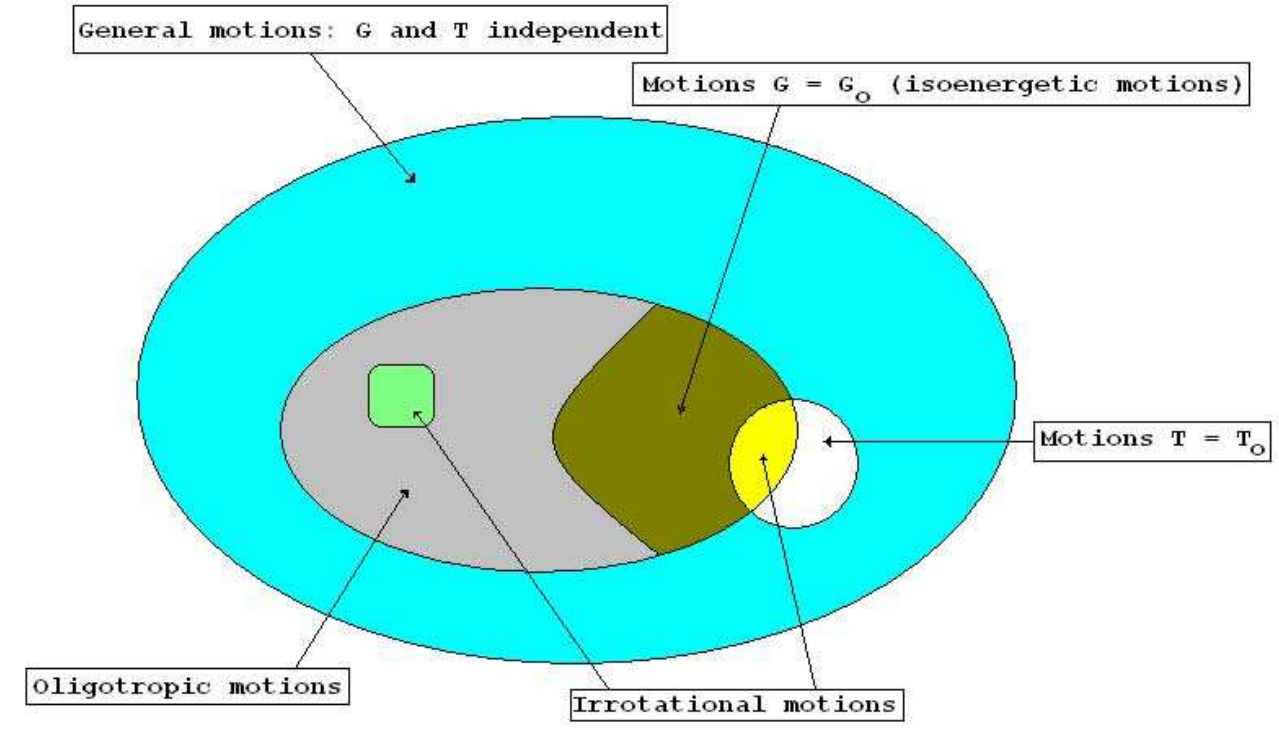

Fig. 2. Classification of permanent motions: the different domains do not mix along the motion of the fluid.

(c) $G$ is constant and $T$ is not constant.

This is a particular case of $(\mathrm{b}):\left(\frac{d \psi}{d t}=u\right)$.

(d) $T$ is constant, $G$ is not constant. The equations are

$$
\left\{\begin{aligned}
\mathbf{V} & =\operatorname{grad} \varphi+\gamma \operatorname{grad} G \\
\frac{d \gamma}{d t} & =-1, \frac{d G}{d t}=0, \quad T=T_{o}
\end{aligned}\right.
$$

(e) $T$ and $G$ are constant.

We obtain the irrotational motions

$$
\mathbf{V}=\operatorname{grad} \varphi, G=G_{o}, T=T_{o}
$$

Let us note: All the irrotational motions are not represented by case (e) (such is the case of Hamel's motions [26]). In fact, relation (16) points out that if the motion is irrotational and if one of the mappings $T$ or $G$ is constant, the other mapping is also constant. If neither $G$ nor $T$ are constant, $G$ must depend on $T$ (case (b)): an irrotational motion is oligotropic.

Irrotational motions do not correspond to particular values of the first integrals 
(or values convected by the flow). In Fig. 2, we represent the flow of a fluid with different domains in $\mathcal{D}_{t}$. The domains are convected by the flow and do not overlap. If each domain has its own color, it keeps the color all along the motion.

\section{Appendix}

The motion of the medium consists of the $t$-dependent $C^{2}$-diffeomorphism $\varphi_{t}[5]$

$$
\mathrm{X} \in \mathcal{D}_{o} \longrightarrow \mathbf{x} \in \mathcal{D}_{t}
$$

We deduce $\frac{d F}{d t}=\frac{\partial \mathbf{V}}{\partial \mathbf{x}} F$ and $\frac{d F^{-1}}{d t}=-F^{-1} \frac{\partial \mathbf{V}}{\partial \mathbf{x}}$.

Let us write $T^{*}\left(\mathcal{D}_{t}\right)$ for the cotangent fiber bundle of $\mathcal{D}_{t}$ and $T_{\mathbf{x}}^{*}\left(\mathcal{D}_{t}\right)$ for the cotangent linear space to $\mathcal{D}_{t}$ at $\mathbf{x}$; then,

$$
\mathbf{x} \in \mathcal{D}_{t} \longrightarrow L(\mathbf{x}, t) \in T_{\mathbf{x}}^{*}\left(\mathcal{D}_{t}\right)
$$

represents a field of differential forms on $\mathcal{D}_{t}$.

Let us write $T^{*}\left(\mathcal{D}_{o}\right)$ the cotangent fiber bundle of $\mathcal{D}_{o}$ and $T_{\mathbf{X}}^{*}\left(\mathcal{D}_{o}\right)$ the cotangent linear space to $\mathcal{D}_{o}$ at $\mathbf{X}$. The mapping $\varphi_{t}^{*}$ is induced by $\varphi_{t}$ for the form fields. The convective derivation $d_{c}$ of a form field $L$ is deduced from the diagram

$$
\begin{array}{ccc}
L \in T^{*}\left(\mathcal{D}_{t}\right) & \stackrel{\varphi_{t}^{-1 *}}{\longrightarrow} & L F \in T^{*}\left(\mathcal{D}_{o}\right) \\
d_{c} \downarrow & \downarrow \frac{d}{d t} \\
\frac{d L}{d t}+L \frac{\partial \mathbf{V}}{\partial x} & \longleftarrow & \frac{d L}{d t} F+L \frac{\partial \mathbf{V}}{\partial x} F \in T^{*}\left(\mathcal{D}_{o}\right)
\end{array}
$$

Let us note that $\frac{d L}{d t}+L \frac{\partial \mathbf{V}}{\partial \mathbf{x}}$ is the Lie derivative of $L$ with respect to the velocity field $\mathbf{V}^{6}$.

The velocity field $\mathbf{V}$ is the infinitesimal transformation of the one-parameter group of transformation $\varphi_{t}[27]$.

${ }^{6}$ Generally, for any tensor, the convective derivative is the Lie derivative with respect to the velocity field. 
Consequences : $\mathcal{D}_{t}$ is assumed to be an Euclidian space; let $b$ be a scalar field on $\mathcal{D}_{t}$. We define two form-fields as $\mathbf{V}^{*}$ and $(\operatorname{grad} b)^{*}$. From $(20)$ we deduce

$$
d_{c}\left(\mathbf{V}^{*}\right)=\Gamma^{*}+\frac{\partial}{\partial x}\left(\frac{1}{2} \mathbf{V}^{2}\right)
$$

where $\boldsymbol{\Gamma}$ is the acceleration vector. Taking into account that

$$
d_{c}(\operatorname{grad} b)^{*}=\left(\operatorname{grad} \frac{d b}{d t}\right)^{*} \text { and } d_{c}\left(L F^{-1}\right)=\frac{d L}{d t} F^{-1},
$$

Eq. (3) yields

$$
d_{c} \mathbf{V}^{*}=\operatorname{grad}^{*}\left(\frac{1}{2} \mathbf{V}^{2}-g-\Omega\right)+\theta \operatorname{grad}^{*} s .
$$

Lemma 1 Let $\theta$ and $s$ be two differential scalars fields in $\mathcal{D}_{t}$.

There exist three scalar fields $\alpha, \beta, \gamma$ such that

$$
\theta \operatorname{grad}^{*} s=\alpha \operatorname{grad}^{*} \beta+\operatorname{grad}^{*} \gamma
$$

is equivalent to: there exists a function $\varphi$ such that

$$
\beta=\varphi(\theta, s)
$$

Proof :

- If $\operatorname{grad} \theta \wedge \operatorname{grad} s=0$ then $\theta=\Phi^{\prime}(s), \theta \operatorname{grad} s=\operatorname{grad} \Phi(s)$ and $\beta=0$.

- If on an open set, $\operatorname{grad} \theta \wedge \operatorname{grad} s \neq 0$ then

$$
(i) \Rightarrow \operatorname{grad} \theta \wedge \operatorname{grad} s=\operatorname{grad} \alpha \wedge \operatorname{grad} \beta
$$

Consequently, $\operatorname{grad} \theta, \operatorname{grad} s, \operatorname{grad} \alpha$ and $\operatorname{grad} \beta$ are in the same plane and there exist $\varphi, \psi, \chi$ such that $\beta=\varphi(\theta, s), \alpha=\psi(\theta, s)$ and $\gamma=\chi(\theta, s)$. So, $(i) \Rightarrow(i i)$.

(ii) yields locally $\theta=h(\beta, s)$.

There exists $\gamma=H(\beta, s)$ such that $H_{s}^{\prime}=\theta$. With $\alpha=-H_{\beta}^{\prime}$, we obtain $\operatorname{grad}^{*} \gamma=H_{s}^{\prime} \operatorname{grad}^{*} s+H_{\beta}^{\prime} \operatorname{grad}^{*} \beta$. So, $(i i) \Rightarrow(i)$.

Lemma 2 Let $\sigma_{o}$ be an arbitrary non-constant differential scalar field in $\mathcal{D}_{o}$ independent of a given scalar field $\delta_{o}$ defined on $\mathcal{D}_{o}$ by the relation $\delta_{o}(\mathbf{X})=$ $\delta\left[\varphi_{t}(\mathbf{X})\right]$. For any form field $\mathbf{X} \in \mathcal{D}_{o} \longrightarrow \Xi_{o}^{*}(\mathbf{X}) \in T_{X}^{*}\left(\mathcal{D}_{o}\right)$ there exist -at least locally- three scalar fields defined on $\mathcal{D}_{o}$ and denoted by $\alpha_{o}, \beta_{o}$ and $\omega_{o}$ such that

$$
\Xi_{o}^{*}=\operatorname{grad}_{o}^{*} \alpha_{o}+\beta_{o} \operatorname{grad}_{o}^{*} \delta_{o}+\omega_{o} \operatorname{grad}_{o}^{*} \sigma_{o}
$$


Due to the fact that $\operatorname{grad}_{o}^{*} \delta_{o}$ and $\operatorname{grad}_{o}^{*} \sigma_{o}$ are two independent forms of $\mathcal{D}_{o}$, the two surface-families $\delta_{o}(X)=a_{1}$ and $\sigma_{o}(X)=b_{1}$ (where $a_{1}$ and $b_{1}$ are two arbitrary constants), generate a curve family denoted by $(\Gamma)_{a_{1} b_{1}}$.

Let $(S)$ be any transverse surface of the curve family $(\Gamma)_{a_{1} b_{1}}$, point $M_{o}$ denotes the current intersection between $(\Gamma)_{a_{1} b_{1}}$ and $(S)$. We denote by $\widetilde{M_{o}} M$ the arc of the curve $(\Gamma)_{a_{1} b_{1}}$ with origin at $M_{o}$.

We define a differential function as

$$
\mathbf{X} \in \mathcal{D}_{o} \longrightarrow \alpha_{o}(\mathbf{X})=\int_{M_{o} M} \Xi_{o}^{*} d \mathbf{X}
$$

such that $d \delta_{o}=0, d \sigma_{o}=0$ and $d \alpha_{o}=\Xi_{o}^{*} d \mathbf{X}$. Then, there exist -at least locally- two scalar field forms $\beta_{o}$ and $\omega_{o}$ in $\mathcal{D}_{o}$ such that

$$
\Xi_{o}^{*} d \mathbf{X}-d \alpha_{o}=\beta_{o} d \delta_{o}+\omega_{o} d \sigma_{o}
$$

Theorem 3 For any mapping $\sigma$ of $\mathcal{D}_{t}$, independent of $\delta$ and verifying $\frac{d \sigma}{d t}=0$, for any form field $\Xi^{*}$ in $\mathcal{D}_{t}$ with a zero convective derivation, there exist-at least locally- three mappings $\alpha, \beta$ and $\omega$ of $\mathcal{D}_{t}$, verifying $\frac{d \alpha}{d t}=0, \frac{d \beta}{d t}=0$ and $\frac{d \omega}{d t}=0$ such that $\Xi^{*}=\operatorname{grad}^{*} \alpha+\beta \operatorname{grad}^{*} \delta+\omega \operatorname{grad}^{*} \sigma$.

Let us consider $\sigma(t, \mathbf{x})=\sigma_{o}\left[\varphi_{t}^{-1}(\mathbf{X})\right], \alpha(t, \mathbf{x})=\alpha_{o}\left[\varphi_{t}^{-1}(\mathbf{X})\right], \beta(t, \mathbf{x})=\beta_{o}\left[\varphi_{t}^{-1}(\mathbf{X})\right]$ and the field $\Xi^{*}$ of forms in $\mathcal{D}_{t}$ corresponding to $\Xi_{o}^{*}$.

Since $\sigma, \alpha, \beta, \omega$ and $\delta$ have zero material derivatives, the previous forms and scalar functions have a zero convective derivatives.

Consequences:

From the representation of the form field $\mathbf{C}^{*}$ and for $\delta=T$, we can get two scalar fields $\varphi$ and $\psi$ in System (10); we obtain the potential representation (or Clebsch's representation) for the flows in System (12). We note that the choice of $\mathbf{C}^{*}$ is arbitrary and $\varphi$ and $\psi$ are not unique.

Proof of system (18):

System (18) is directly obtained when $G$ is independent of $T$. In the converse, only a modification is needed: for a given form field $\Xi_{o}^{*}$ in $\mathcal{D}_{o}$, there exist -at least locally- two scalar fields $\alpha_{o}$ and $\beta_{o}$ such that

$$
\Xi_{o}^{*}=\operatorname{grad}_{o}^{*} \alpha_{o}+\beta_{o} \operatorname{grad}_{o}^{*} T_{o}
$$

It is an analog representation as Clesch's [16]. In this case, we consider a transverse curve $(\Gamma)$ of $T$-surfaces and a scalar field

$$
\alpha_{o}(\mathbf{X})=\int_{M_{o} M} \Xi_{o}^{*} d \mathbf{X}
$$

defined on $\mathcal{D}_{o}$ as the circulation of $\Xi_{o}^{*}$ on an arbitrary curve on the $T$-surface 
connecting point $\mathbf{X}$ at point $\mathbf{X}_{o}$ where the surface intersects $(\Gamma)$.

The circulation is independent of the curve connecting $\mathbf{X}_{o}$ and $\mathbf{X}$; when $d T_{o}=$ 0 then $d \alpha_{o}=\Xi_{o}^{*} d \mathbf{X}$. Consequently there exists a Lagrangian multiplier $\beta_{o}(\mathbf{X})$ such that

$$
\Xi_{o}^{*} d \mathbf{X}=d \alpha_{o}+\beta_{o} d T_{o}
$$

and the Clebsch form is obtained.

\section{References}

[1] H. Gouin, Comptes rendus Acad. Sci. Paris, 305, II (1987) 833.

[2] M. Barrere and R. Prud'homme, Equations fondamentales de l'aérothermochimie, Masson, Paris (1973).

[3] D.J. Korteweg, Archives Néerlandaises, 28, (1901) 1.

[4] J. Serrin, Ed., New Perspectives in Thermodynamics, Springer, New York (1986).

[5] J. Serrin, Encyclopedia of Physics VIII/1, Springer, Berlin (1959).

[6] P. Casal, J. de Mécanique, 5, 2 (1966) 149.

[7] H. Gouin, J. de Mécanique, 20, 2 (1981) 273.

[8] P. Casal and H. Gouin, Sur les interfaces liquide-vapeur non-isothermes, J. de Mécanique Théorique et Appliquée, 7, 6 (1988) 689.

[9] P. Casal and H. Gouin, A representation of liquid-vapour interfaces by using fluids of second grade, Annales de Physique, 13, 3 coll. 2(1988) 3.

[10] R.L. Seliger and G.B. Whitham, Proc. Roy. Soc. of London, A, 305 (1968) 1.

[11] H. Gouin, Research Notes in Mathematics, 46, Pitman, London (1981) 128.

[12] P. Casal and H. Gouin, Comptes rendus Acad. Sci. Paris, 306, II (1988) 99.

[13] F. Cubisol, Contribution à une étude thermomécanique des milieux continus, thesis, Université d'Aix-Marseille (1987).

[14] C. Truesdell and R.A. Toupin, The Classical Field Theories, Encyclopedia of Physics III/1, Springer, Berlin (1960).

[15] C. Truesdell and W. Noll, Encyclopedia of Physics III/3, Springer, Berlin (1965).

[16] H. Lamb, Hydrodynamics, Dover Publications, New York (1972).

[17] L. Landau and E. Lifchitz, Fluid Mechanics, Mir, Moscow (1971). 
[18] F.A. Goldsworthy J. Fluid Mech., 5, (1959) 164.

[19] B. Sturtevant and E. Slachmuyders, Phys. Fluids, 7, (1964) 1201.

[20] J. Mandel, Cours de mécanique des milieux continus, Gauthier-Villars, Paris (1966) 135.

[21] H. Gouin, Mech. Res. Comm., 3, (1976) 151.

[22] J.J. Moreau, Sur les intégrales premières de la dynamique d'un fluide parfait barotrope et le théorème de Helmholtz-Kelvin, Séminaire d'analyse convexe, $\mathbf{3}$, Montpellier (1977).

[23] E. Cartan, Leçons sur les invariants intégraux, Hermann, Paris (1971).

[24] H. Gouin, Contribution à une étude géométrique et variationnelle des milieux continus, Thesis, Université de Provence (1978).

[25] H. Villat, Leçons sur la théorie des tourbillons, Masson, Paris (1930).

[26] G. Hamel, Sitzgsber. preus. Akad. Wiss. Phys. Math. Kl. (1937) 5.

[27] P. Germain, Cours de mécanique des milieux continus, Masson, Paris (1973). 